\title{
EVALUACIÓN FORMATIVA Y COMPARTIDA EN EL USO DE EDUBLOGS PARA AUMENTAR LA PARTICIPACIÓN DEL ALUMNADO EN SUS PROCESOS DE ENSEÑANZA-APRENDIZAJE
}

Formative and shared assessment in weblogs' use to increase students’ participation in their teaching-learning process

Avaliação formativa e compartilhada em uso edublogs para aumentar a participação dos estudantes no processo de ensino-aprendizagem

Este estudio se ha llevado a cabo bajo la financiación de los proyectos de innovación educativa de la Universitat de València "Edublogs y aprendizaje colaborativo: concreción de estrategias de enseñanza para el profesorado(UVSFPIE_FO13-147376)" y "Edublogs y aprendizaje colaborativo: concreción de estrategias de evaluación formativa basadas en la coevaluación y en la autoevaluación del alumnado (UV-SFPIE_FO14-223314)" y dentro del proyecto $\mathrm{I}+\mathrm{D}+\mathrm{i}$ "La competencias docentes en la formación inicial del profesorado de educación física (EDU 2013-42024-R)”. Convocatoria de noviembre de 2013 del Programa Estatal de Investigación, Desarrollo e Innovación Orientada a los Retos de la Sociedad, en el marco del Plan Estatal de Investigación Científica y Técnica y de Innovación 2013-2016

\section{Alexandra Valencia-Peris (1) \\ Jorge Lizandra (2) \\ Daniel Martos Garcia (3)}

(1) Universitat de València, España. Teléfono: +34 963828920. Correo electrónico: alexandra.valencia@uv.es

(2) Universitat de València, España. Teléfono: +34 961625094. Correo electrónico: jorge.lizandra@uv.es

(3) Universitat de València, España. Teléfono: +34 963983383. Correo electrónico: daniel.martos@uv.es

\section{Resumen}

El objetivo de este trabajo fue mostrar el grado de participación del alumnado en las diversas experiencias educativas llevadas a la práctica por profesorado perteneciente a Evaluación formativa y compartida en el uso de edublogs para aumentar la participación del alumnado en sus procesos de enseñanza-aprendizaje 
un grupo de innovación educativa relacionadas con la evaluación formativa a través del uso de edublogs. Para ello se analizaron 27 acciones formativas distintas en las que participaron 2303 estudiantes durante los cursos 13/14 y 14/15. El porcentaje de participación del alumnado aumenta conforme lo hace la interactividad de los diferentes tipos de blog y según se otorgue mayor protagonismo a éste en su evaluación. En conclusión, los edublogs son un recurso útil y práctico para aumentar la participación e implicación del alumnado en su proceso de enseñanza-aprendizaje, aunque no siempre se llevan a cabo procesos reales de evaluación formativa y compartida, ni se tiene en cuenta a la hora de calificar al alumnado.

Palabras clave: evaluación formativa, innovación, edublogs

\begin{abstract}
The aim of this work was to show the degree of students' participation in some educational experiences carried out by teachers belonging to an educational innovation group related to formative assessment through weblogs. For this purpose, 27 different educative actions were analyzed in which 2303 students participated during the courses 13/14 and 14/15. The percentage of students' participation increased as the interactivity of the different types of blog does and according to which it gives greater prominence to the latter in its evaluation. In conclusion, weblogs are a useful and practical resource to increase the participation and involvement of students in their teaching-learning process, although real processes of formative and shared assessment are not always accurately carried out and nor are taken into account when qualifying students.
\end{abstract}

Keywords: formative assessment, innovation, weblogs

\title{
Resumo
}

O objetivo deste trabalho foi mostrar o grau de participação dos alunos em diversas experiências educativas postas em prática por professores pertencentes a um grupo de inovação educativa relacionada com a avaliação formativa através do uso de edublogs. Para fazer 27 atividades de formação diferentes em que 2303 alunos participaram dos cursos de 13/14 e 14/15 foram analisadas. O percentual de participação dos alunos aumenta à medida que faz a interação de diferentes tipos de blog e de acordo com o 
maior destaque é dado a ele na sua avaliação. Em conclusão, os edublogs são um recurso útil e prático para aumentar a participação e envolvimento dos alunos no processo ensino-aprendizagem, mas nem sempre são levados a cabo processos reais de formação e avaliação compartilhada e tidos em conta na qualificar os alunos.

Palavras-chave: avaliação formativa, inovação, edublogs

\section{Introducción}

El blog es un recurso ampliamente utilizado en el ámbito de la Educación Superior, en sus diferentes ramas de conocimiento y en el contexto nacional e internacional (Sim \& Hew, 2010). El tipo de blog educativo puede clasificarse en función del grado de participación e interactividad que posibilita, existiendo cinco subtipos. Asimismo, los edublogs son herramientas que facilitan procesos de evaluación y retroalimentación entre estudiantes, así como la autoevaluación en la participación (Molina, Valencia-Peris \& Gómez-Gonzalvo, 2016).

El objetivo de este trabajo es mostrar el grado de participación del alumnado en las diversas experiencias educativas llevadas a la práctica por profesorado perteneciente a un grupo de innovación educativa relacionadas con la evaluación formativa a través del uso de edublogs.

\section{Método}

Los datos utilizados son parte de la información proporcionada en los informes semiestructurados sobre la docencia impartida por docentes universitarios que forman parte de un Grupo de Innovación (GID9/2009), que a priori han puesto en práctica un sistema de evaluación formativa y compartida durante los cursos 2013-2014 y 20142015 a través de la utilización de edublogs. Concretamente, recopilamos 27 acciones formativas distintas en las que participaron 2303 estudiantes provenientes de distintas titulaciones (principalmente del Grado en Ciencias de la Actividad Física y el Deporte y del Grado en Educación Primaria) de 6 universidades españolas. Se realizó un análisis de contenido de los 27 informes, basado fundamentalmente en las categorías “Concreción de los usos didácticos que se le ha dado al blog” e "Integración de la participación y uso del edublog en el sistema de evaluación de la asignatura. 


\section{Resultados y discusión}

De los 2303 estudiantes matriculados en las asignaturas estudiadas un 81\% participó en los blogs planteados por el profesorado $(n=1854)$, porcentaje muy superior a los datos apuntados por Molina et al. (2016) en sus experiencias durante cuatro cursos académicos. Tal y como se observa en la Tabla 1, el porcentaje de participación del alumnado en el edublog aumenta conforme asciende el nivel de participación e interactividad que estos proporcionan (Molina, Valenciano \& Valencia-Peris, 2015).

La tabla 2 muestra el porcentaje de participación en función del tipo de evaluación planteada para los estudiantes en 12 de los 27 edublogs (un 44,4\%). Siguiendo los argumentos sobre evaluación de López Pastor (2009), se observa que aquéllos blogs en los que se ofrecen procesos de evaluación más democráticos como la autoevaluación y la co-evaluación obtienen porcentajes más elevados de participación que aquellos que se basan en una evaluación más tradicional por parte del profesorado. Los blogs que suelen ofrecer una autoevaluación en la participación de los estudiantes son aquellos abiertos a la participación a través de sus comentarios (tipo b). Por el contrario, aquellos que ofrecen la co-evaluación son experiencias donde se han llevado a cabo blogosferas educativas (tipo e), las cuales permiten mayor interactividad de sus usuarios/as

Tabla 1.

Participación del alumnado en función del tipo de blog empleado en la asignatura.

\begin{tabular}{cccc}
\hline Tipo de blog & $\begin{array}{c}\text { M alumnos } \\
\text { matriculados }\end{array}$ & $\begin{array}{c}\text { M alumnos que } \\
\text { participan }\end{array}$ & \% de participación \\
\hline a & 103 & 65 & 63 \\
b & 100 & 65 & 65 \\
c & 77 & 71 & 92 \\
d & 49 & 45 & 92 \\
e & 76 & 71 & 93 \\
\hline
\end{tabular}

M: Media; a: El blog docente como transmisor de la información de la asignatura; b: El blog abierto a la participación del alumnado a través de sus comentarios; c: El blog abierto a la participación del alumnado a través de sus posts; d: Los alumnos como administradores de sus blogs; e: Blogosferas.

Tabla 2.

Participación del alumnado en función del tipo de evaluación realizada en el edublog.

\begin{tabular}{lccc}
\hline \multicolumn{1}{c}{ Tipo de evaluación } & $\begin{array}{c}\text { M alumnos } \\
\text { matriculados }\end{array}$ & $\begin{array}{c}\text { M alumnos que } \\
\text { participan }\end{array}$ & \% de participación \\
\hline Autoevaluación & 67 & 64 & 96 \\
Co-evaluación & 108 & 77 & 77 \\
Heteroevaluación & 80 & 59 & 74 \\
\hline
\end{tabular}

M: Media

Evaluación formativa y compartida en el uso de edublogs para aumentar la participación del alumnado en sus procesos de enseñanza-aprendizaje 
Asimismo, los porcentajes de evaluación correspondientes a la participación en los edublogs oscilaron entre el 0.3 y el $60 \%$ de la nota final, considerándose en algunos de ellos incluso voluntaria. Este hecho muestra que pese a incluir estos instrumentos en el proceso de enseñanza-aprendizaje de las asignaturas, el profesorado aún se muestra reticente a la hora de contabilizarlos para calificar al alumnado. En este sentido, el no sentirse lo suficientemente competentes para otorgarle a los edublogs un papel evaluador de los procesos de enseñanza-aprendizaje puede ser uno de los motivos por los cuales un 25,9\% del profesorado no les da ningún peso en la evaluación (Gallego, Gámiz \& Gutiérrez, 2010). Normalmente estas experiencias han utilizado el blog como tablón de anuncios y distribuidor de información para el alumnado (tipo a).

\section{Conclusiones}

Los edublogs son un recurso útil y práctico para aumentar la participación y la involucración del alumnado en su proceso de enseñanza-aprendizaje. Pese a ello, son pocas las experiencias de innovación con edublogs que han tenido en cuenta procesos reales de evaluación formativa y compartida con un peso real en la calificación final de la asignatura. A tenor de los resultados de este estudio cabe mejorar la competencia digital del profesorado universitario para dotar de uso pedagógico a las TIC y lograr su integración real en los procesos de enseñanza-aprendizaje y por ende en los sistemas de evaluación.

\section{Referencias}

Gallego, M.J., Gámiz, V., \& Gutiérrez, E. (2010). El futuro docente ante las competencias en el uso de las tecnologías de la información y comunicación para enseñar. EDUTEC. Revista Electrónica de Tecnología Educativa, 34, 1-18.

López Pastor, V.M. (2009). Fundamentación teórica y revisión del estado de la cuestión. En V.M. López Pastor (coord.), Evaluación Formativa y Compartida en Educación Superior. Propuestas, técnicas, instrumentos y experiencias (pp. 4564). Madrid: Narcea Ediciones.

Molina, P., Valenciano, J., \& Valencia-Peris, A. (2015). Los blogs como entornos virtuales de enseñanza y aprendizaje en Educación Superior. Revista Complutense de Educación, 26(esp), 15-31. 
Molina, P., Valencia-Peris, A., \& Gómez-Gonzalvo, F. (2016). Innovación docente en Educación Superior: Edublogs, evaluación formativa y aprendizaje colaborativo. Profesorado. Revista de currículum y formación del profesorado, 20(2), 432-449.

Sim, J.W.S., \& Hew, K.F. (2010). The use of weblogs in higher education settings: A review of empirical research. Educational Research Review, 5(2), 151-163.

Evaluación formativa y compartida en el uso de edublogs para aumentar la participación del alumnado en sus procesos de enseñanza-aprendizaje 